\title{
Une étude du crédit aux éleveurs de P. Lhostc 1 bovins au Cameroun
}

LHOSTE (P.). Une étude du crédit aux éleveurs de bovins au Cameroun. Revue Élev. Méd. vét. Pays trop., 1990, 43(1):111-117.

Un programme de crédit aux éleveurs bovins traditionnels du Cameroun a été mis en place de 1975 à 1986 pour favoriser la modernisation de ce secteur. Une évaluation effectuée en 1986 a mis en évidence les difficultés rencontrées par ce projet qui n'a pas vraiment atteint les objectif́s escomptés. Il apparaît essentiel de préciser le statut juridique des terres de pâturage et de responsabiliser les communautés d'éleveurs dans ce type de programme. Mots clés : - Bovin - Éleveur Élevage traditionnel - Crédit - Cameroun.

\section{INTRODUCTION}

Dans le cadre d'une évaluation de la Banque mondiale, une étude sur l'impact d'un programme de crédit à l'élevage bovin a été menée au Cameroun, d'octobre à novembre 1986, dans les provinces de l'Adamaoua (N'Gaoundéré), de l'Ouest (Bafoussam) et du NordOuest (Bamenda). Cette étude a déja fait l'objet d'un rapport plus complet (9). II est utile d'en retracer le contexte $(7,11)$.

La Plan national de développement de l'élevage, couramment appelé "Plan viande ", lancé en 1974-75, comportait plusieurs volets, dont un programme de modernisation de l'élevage traditionnel par le crédit aux éleveurs de bovins, qui s'est étendu sur une dizaine d'années (deux périodes quinquennales) à l'issue desquelles une évaluation était demandée. La présente étude porte sur les modalités, le déroulement et l'impact technique et éconmique de ce programme et avance des propositions pouvant améliorer son fonctionnement et son efficacité au profit des éleveurs et de l'État camerounais.

Un bref rappel est fait des principales caractéristiques des systèmes d'élevage bovin pour les régions étudiées $(2,3)$.

\section{Le système d'élevage bovin traditionnel}

II est encore largement dominant en Adamaoua* et dans l'Ouest; fondé sur une utilisation extensive et presque exclusive des pâturages naturels, une de ses caractéristiques est la mobilité du troupeau:

- mobilité dans un domaine pâturable, souvent collectif, assez vaste ;

- mobilité saisonnière (variable selon les éleveurs) dans le cadre de la transhumance de saison sèche.

II est possible de classer ces élevages selon l'importance des déplacements du troupeau (sédentaire, petite transhumance, grande transhumance...) ; l'organisation sociale de l'élevage (qui évolue rapidement), et notamment le statut de l'éleveur, apparaît aussi comme un critère d'analyse essentiel. En effet, il existe une grande diversité entre des éleveurs stricts vivant avec leur troupeau, des propriétaires d'animaux pluriactifs habitant sur place et gérant leur troupeau avec l'aide de bouviers salariés et des propriétaires éloignés qui s'en remettent à des "représentants ". II semble que la propriété du cheptel bovin ait tendance à se déplacer des éleveurs authentiques vers les éleveurs pluriactifs, et l'action du crédit n'est pas négligeable dans un tel processus.

\section{L'élevage bovin associé à l'agriculture}

Ce système est surtout représenté dans l'Ouest et le Nord-Ouest (où il est appelé mixed-farming), mais il existe de nombreux stades intermédiaires avec le système extensif décrit précédemment. Cette fois, les effectifs bovins sont plus faibles et constitués d'animaux de trait, de petits lots d'embouche ou de quelques vaches laitières ou allaitantes. Les relations avec l'exploitation agricole sont plus fortes, avec une intégration se traduisant par la stabulation et l'affouragement des animaux. Ce système se prête bien à l'intensification et à une amélioration des complémentarités agriculture-élevage, notamment par l'utilisation de l'énergie animale, la valorisation de la fumure organique et des résidus des cultures.
1. Mission IEMVT-CIRAD, 9 place Viala, 34060 Montpellier Cedex.

Reçu le 08.09.89, accepté le 12.10.89.
* En Adamaoua, 96 p. 100 de l'effectif bovin relève, de ce système (5). 


\section{L'élevage moderne ou ranching}

$\mathrm{Ce}$ système est fondé sur une utilisation plus rationnelle des pâturages: ajustement des charges, rotations, compléments alimentaires adaptés, etc. L'Adamaoua possède quelques élevages privés qui font une démonstration exemplaire de l'efficacité de ce système d'élevage (notamment la Compagnie pastorale, dont les techniques ont fait leur preuve et peuvent être à juste titre valorisées dans la région). C'est ce système de ranching que souhaite promouvoir le Plan national de développement de l'élevage. A noter que, selon O. DAWA (5), le ranching ne concerne, en Adamaoua, qu'environ 60000 têtes, soit 4 p. 100 de l'effectif.

Les éleveurs bénéficiaires du crédit appartiennent aux deux premiers systèmes décrits.

\section{Les effectifs}

Le cheptel bovin camerounais est estimé à près de 4 millions de têtes. Pour les régions concernées par l'étude, les estimations du Service de l'élevage étaient en 1986 de 1800000 bovins répartis comme suit :

- Adamaoua : 1170000

- Nord-Ouest : 460000

- Ouest : 170000

\section{MÉTHODOLOGIE}

La méthodologie de l'enquête et son traitement ont déjà été développés (9).

Malgré les distances et les problèmes pratiques de rendez-vous, les contacts directs avec les bénéficiaires du crédit ont été priviligiés.

Un tirage au sort a été effectué dans les listes des bénéficiaires, détenues par l'organisme responsable, et 75 éleveurs, sur un ensemble de 956 (soit 8 p. 100), ont été enquêtés. En fait, il n'a pas été possible de contacter tous les éleveurs tirés au sort, pour diverses raisons (décédés, absents, non disponibles...).

\section{L'enquête}

Un guide d'entretien a été préparé et testé auprès de quelques éleveurs, lors d'une pré-enquête, au début de l'étude. Ce guide comportait 11 sections principales plus ou moins détaillées:

1. l'éleveur et sa famille (organisation, actifs...)
2. activités principales (élevage et autres...);

3. main-d'oeuvre

4. localisation (habitation, élevage...)

5. foncier (effectif et officiel, selon dossier de crédit)

6. crédit : modalités, utilisation, remboursement

7. suivi du FONADER

8. aspects zootechniques et sanitaires concernant le troupeau: composition, conduite, alimentation, santé, ventes

9. association agriculture-élevage

10. récapitulation des charges et des revenus

11. opinions générales de l'éleveur.

II est clair qu'une enquête rapide, en un seul passage, ne permettait pas toujours d'obtenir une information détaillée sur ces différents thèmes. Ainsi, les données relatives à la productivité du troupeau sont rares et peu fiables. Pratiquement aucun éleveur ne possède de documents d'élevage utilisables.

Comment évaluer, dans ces conditions, la productivité du troupeau et, surtout, comment en apprécier l'éventuelle variation sous l'effet du crédit? Certaines questions sont donc restées sans réponse précise car il aurait fallu déployer des moyens plus importants pour y répondre.

\section{L'entretien}

Les entretiens ont eu lieu dans des conditions variables, le plus souvent avec un interprète. La situation idéale était de rencontrer l'éleveur sur le terrain pour parler précisement de son troupeau et constater ses réalisations. Cela n'a pas toujours été possible; certains entretiens ont eu lieu à la préfecture, dans une boutique, sur un marché, etc.

\section{Le traitement de l'enquête}

II fait appel,' d'une part, aux méthodes statistiques classiques (moyennes, histogrammes, tests de comparaison, tableaux croisés), et d'autre part à l'analyse factorielle des correspondances. Celle-ci permet, en particulier, de prendre en compte simultanément des variables qualitatives (nombreuses dans ce type d'enquête) et des variables quantitatives.

\section{RÉSULTATS}

Les figures 1,2 et 3 présentent quelques résultats de l'analyse factorielle des correspondances. Dans la 


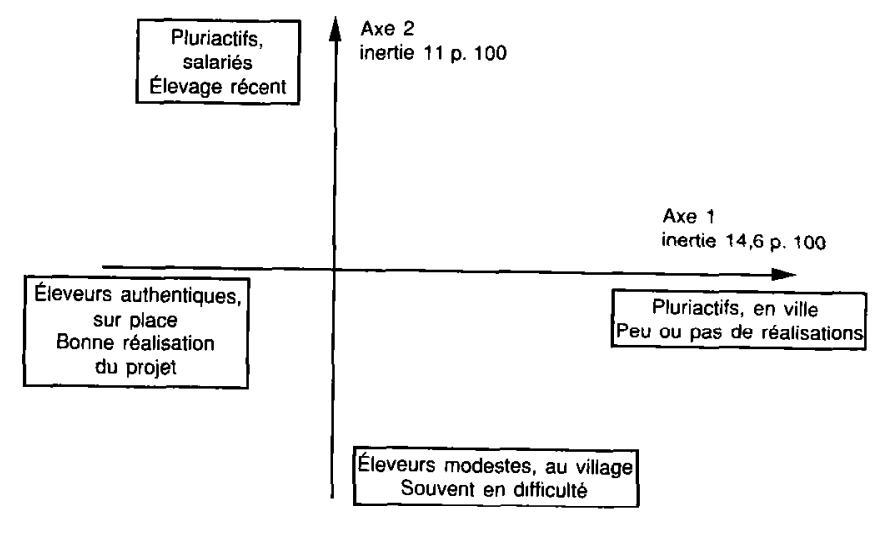

fig. 1

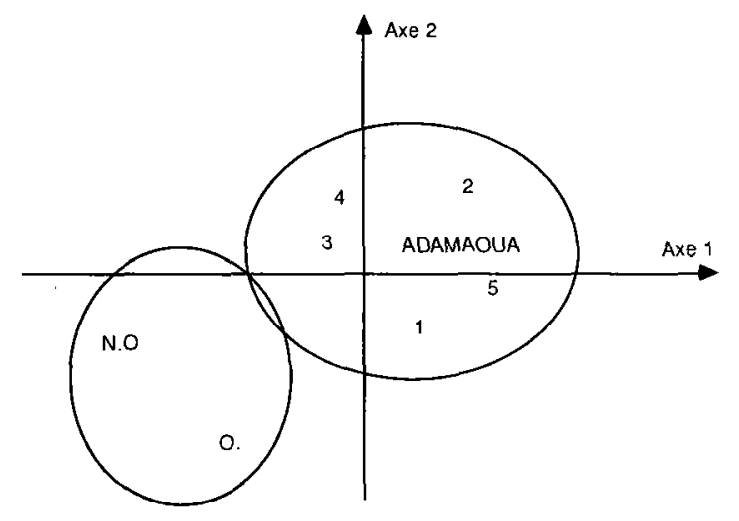

Adamaoua : $1=$ Faro et Deo $; 2=$ Mbéré $; 3=$ Djerem $; 4=$ Vina $; 5=$ Mayo-Banyo N.O. : province du Nord-Ouest $O$. : province de l'Ouest

fig. 2

figure 1, l'axe 1 traduit la réalisation plus ou moins bonne du projet, l'axe 2 tend à opposer les petits éleveurs en difficulté aux pluriactifs salariés. Dans la figure 2, la position des modalités de la variable DEP (département ou province) projetées sur le premier plan factoriel traduit déjà un comportement différent, pour l'ensemble de l'Adamaoua d'une part (DEP 1 a 5), et l'ensemble Ouest et Nord-Ouest d'autre part (DEP 6 et 7).

C'est dans le Nord-Ouest que se rencontre la plus forte proportion d'éleveurs authentiques (M'Bororos notamment) vivant avec leur troupeau. Au contraire, dans les départements de Banyo ou Meiganga, ils sont les moins nombreux (selon l'échantillon). Ce sont également ces éleveurs du Nord-Ouest qui ont tendance à réaliser plus fidèlement le projet d'élevage, en relative conformité avec l'étude technique.

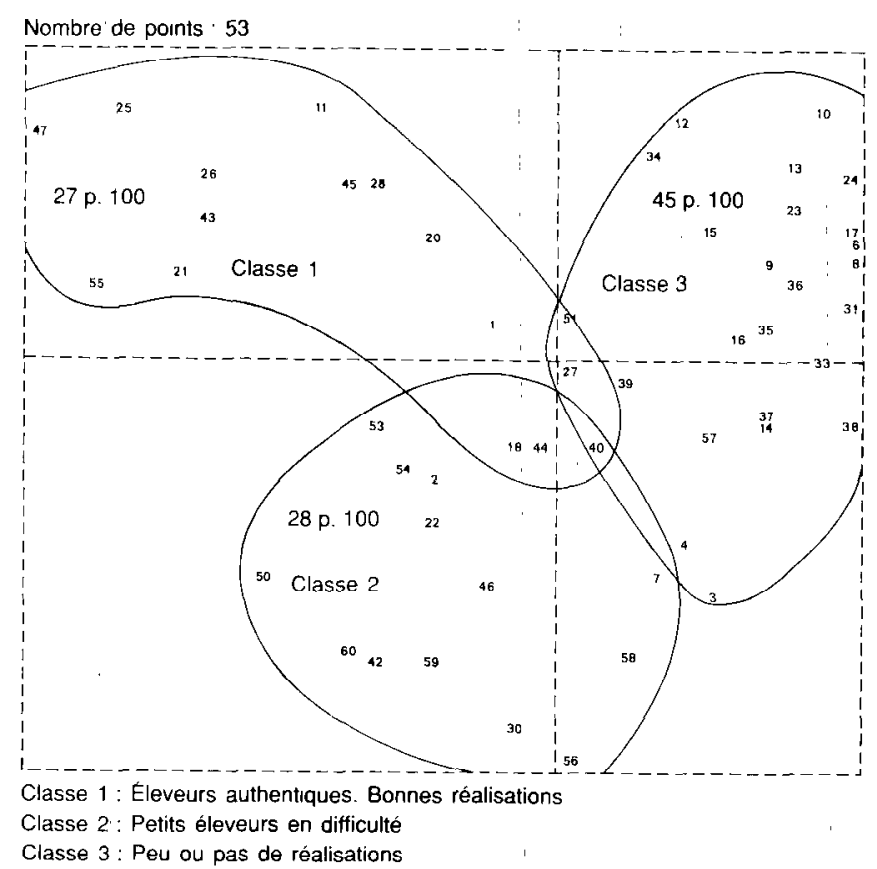

fig. 3

La figure 3 présente une classification des éleveurs qui fait apparaître trois groupes principaux définis ainsi :

\section{Groupe 1 : éleveurs authentiques (27 p. 100)}

II s'agit plutôt de grands troupeaux; l'exécution du projet est relativement correcte.

\section{Groupe 2 : petits éleveurs en difficulté (28 p. 100)}

Ce sont parfois de nouveaux éleveurs qui ont mal réalisé leur projet et qui ont un troupeau de taille modeste (ou pas de troupeau du tout !). Ils rencontrent en général de grandes difficultés de remboursement.

\section{Groupe 3: peu ou pas de réalisation du projet (45 p. 100)}

II correspond souvent à des pluriactifs dont certains sont devenus éleveurs grâce au crédit (fonctionnaires par exemple). Les uns ont bien constitué un troupeau, sans se conformer nécessairement aux termes de l'étude technique; d'autres n'ont réalisé aucun projet d'élevage.

Ces résultats, qui pourraient paraître pessimistes, sont confirmés par d'autres sondages comme celui d'une Commission de contrôle (4) qui estime pour le Mbéré (Meiganga), après avoir visité 51 ranchs, que:

- 2 sont modernes, c'est-à-dire conformes au projet ;

- 18 sont viables (peuvent être maintenus) ; 
- 31 sont fictifs, c'est-à-dire sans réalisation sur le terrain.

Le délégué provincial de l'élevage en Adamaoua confirme que les résultats ne sont pas satisfaisants et que les conséquences de ce programme peuvent être graves et néfastes: "On constate cependant que le ranching, introduit il y a quelques années dans cette Province, en vue de la modernisation de l'activité pastorale pour une meilleure production de viande, n'a pas donné les résultats escomptés et tend même de plus en plus à mettro en péril l'avenir de l'élevage en Adamaoua." (5).

\section{DISCUSSION}

L'étude a permis de mettre en évidence les difficultés rencontrées dans l'application du programme de crédit aux éleveurs. Elles tiennent à plusieurs causes exposées ici par ordre d'importance.

\section{L'imprécision du statut juridique des terres de pâturage}

Dans les faits, l'attribution de terres aux bénéficiaires de prêts a posé de nombreux problèmes et a fait l'objet de divers abus: surfaces octroyées arbitraires et excessives, clôtures réalisées par certains sans rapport avec le droit de pâturage obtenu, droits coutumiers bafoués (points d'eau, pistes à bétail, zones de transhumance, etc.), mêmes terres attribuées à plusieurs reprises.

Deux types principaux d'irrégularités sont constatés :

- l'appropriation d'un domaine spatial excessif (par certains, pas toujours éleveurs, rarement traditionnels) avec une couverture juridique approximative ;

- l'attribution fictive de terrains qui ne seront ni aménagés, ni même, dans certains cas, occupés par le troupeau.

C'est évidemment le premier comportement cité qui a parfois posé de très sérieux problèmes sur le terrain, comme le souligne le délégué provincial de l'élevage en Adamaoua: "Mais le problème le plus fondamental à l'heure actuelle (1986) réside dans l'accaparement des terres par un petit nombre d'éleveurs pour un petit nombre d'animaux au détriment du reste des éleveurs du secteur traditionnel...".. "Les conséquences sont aujourd'hui celles que nous connaissons, c'est-à-dire que le ranching est devenu une occasion pour les riches d'accaparer toutes les bonnes terres au détri- ment des pauvres du secteur traditionnel, plus nombreux et vers qui toutes les actions de modernisation devraient être orientées... »

Ce constat plutôt négatif, sur lequel s'accordent divers observateurs $(5,6,10)$ attentifs de l'élevage au Cameroun, a des causes profondes. Certaines relèvent des procédures sommaires utilisées, d'autres sont liées au manque de dispositions juridiques claires pour l'attribution des droits de pâturage (Adamaoua), des grazing permit et map au Nord-Ouest ; il s'agit de documents officiels revêtus des signatures engageant l'administration (sous-préfet), les autorités traditionnelles (Lamido en Adamaoua, Ardo et Fon au Nordouest) et le Service de l'élevage. En réalité, ces documents ne constituent pas des titres fonciers et les éleveurs n'en usent plus, dans la plupart des cas, pour poser des clôtures et délimiter effectivement leur territoire; ils apparaissent plutôt, dans les faits, comme un passe-droit contraignant exigible pour l'obtention d'un prêt. Les superficies attribuées dans ces documents officiels sont souvent irréalistes; l'interprétation des droits réels conférés par ces documents est l'objet de maintes controverses.

\section{L'imprécision dans la cible du crédit}

Les documents officiels $d u$ «Plan viande » camerounais sont clairs sur l'objectif prioritaire de modernisation des petites unités familiales de l'élevage traditionnel. En Adamaoua, il s'agissait de promouvoir la mise en place de ranchs privés d'environ 250 ha correspondant à des troupeaux familiaux de 50 à 60 têtes. Dans la pratique, ce sont des unités d'environ 750 ha en moyenne qui ont été substituées à celles, trois fois plus petites, du projet initial. Des ranchs privés encore plus grands se sont aussi constitués, au profit de personnages déjà puissants qui ont eu ainsi un accès officiel au foncier.

Le projet, détourné de son objectif et de sa cible, a donc permis, dans certains cas, d'officialiser l'accaparement de grandes portions d'espace par des éleveurs non traditionnels.

\section{L'insuffisance des propositons techniques}

Le modèle technique sur lequel se fonde l'organisme de crédit est pratiquement unique (10). II préconise :

- la mise en place de clôtures (barbelés), de pistes et de pare-feu ;

- l'aménagement de points d'abreuvement, de mangeoires, de corrals ; 
- l'achat de matériel de pulvérisation, de médicaments, d'aliments pour le bétail ;

\section{- l'achat d'animaux.}

Ce modèle n'est pas réaliste pour la majorité des éleveurs concernés: la clôture est encore trop coûteuse pour ce système d'élevage, et elle pose les problèmes déja évoqués, liés au foncier; les pare-feu individuels ne peuvent être efficaces et rentables que si le contrôle des feux devient un objectif de la comunauté entière; certains investissements (pistes, points d'eau, bains détiqueurs...) doivent être envisagés au niveau d'un groupe d'éleveurs. Dans les conditions effectives du crédit, ils se sont révélés beaucoup trop coûteux pour l'éleveur individuel.

II faut donc envisager des formules plus diversifiées et mieux adaptées aux projets individuels des éleveurs.

Dans les conditions du crédit des années 1976-1986, il apparaît que de nombreux éleveurs, qui avaient suivi les recommandations de l'étude technique, ont eu les plus grandes difficultés à faire face à leur endettement. Au contraire, ceux qui ont opté pour un maximum de bétail et un minimum d'investissement (ne respectant donc pas les propositions de l'étude technique) s'en sont beaucoup mieux sortis. Apparemment, le troupeau bovin, bien géré, peut supporter un crédit à 10 p. 100 (certains éleveurs en ont fait la preuve) alors que l'équipement d'un ranch moderne justifie un crédit moins cher pour être rentable.

\section{Les modalités du crédit}

Cet aspect du projet a posé de gros problèmes pratiques pour de nombreux éleveurs traditionnels qui auraient dû être la cible privilégiée du crédit. C'est en fait un problème classique en milieu rural africain (8).

Les deux contraintes majeures inhérentes aux procédures utilisées semblent être:

- l'excessive lourdeur des dossiers ;

- l'insuffisante concertation avec la communauté des éleveurs concernés, pour la prise en compte des droits coutumiers.

\section{QUELQUES ORIENTATIONS NOUVELLES}

Il est nettement apparu, au terme de l'étude, que le crédit aux éleveurs bovins, pratiqué au Cameroun depuis une dizaine d'années, n'a pas obtenu tous les résultats escomptés.
Dans son principe, le crédit aux éleveurs bovins est important car il peut constituer un puissant facteur d'évolution de l'élevage; il représente, en effet, l'un des outils de la politique agricole (volet élevage) qui peuvent orienter et stimuler des actions techniques conduisant à une modification des systèmes d'élevage bovin.

Dans ses modalités, le fonctionnement du crédit aux éleveurs bovins devrait être amélioré dans le sens d'un assouplissement des procédures, d'un allégement des dossiers et d'une diversification des propositions; ces améliorations doivent s'accompagner de plus de rigueur et de précision' dans la sélection des éleveurs bénéficiaires. L'idée est de rendre le crédit effectivement accessible aux vrais éleveurs et aux plus motivés. Certes, l'organisme qui gère le crédit a besoin de garanties administratives et financières; la tentation était donc grande de multiplier les pièces du dossier destinées à limiter les risques, mais ce type de dérive technocratique est contradictoire avec l'objectif essentiel du programme. II semble préférable de rechercher plutôt un engagement solidaire des éleveurs en tant que communauté responsable de la gestion de l'espace, associée à la sélection des bénéficiaires, et concernée par les engagements financiers contractés. Cela suppose également une évolution des mentalités chez certains éleveurs.

Diversifier les propositions paraît essentiel: en premier lieu, il faut, au plan technique, adapter les projets de développement appuyés par le crédit à la diversité des aspirations et des conditions réelles des éleveurs; il faut, de plus, au plan financier, considérer que toute action d'amélioration ne doit pas nécessairement passer par le seul canal du prêt (à taux fixe) aux éleveurs privés.

Le crédit aux éleveurs bovins n'est, évidemment, pas une opération qui peut être totalement dissociée d'autres questions d'organisation générale qui conditionnent son efficacité. L'une des causes principales des difficultés rencontrées est liée à l'imprécision du statut juridique des pâturages, laquelle a été la source de problèmes graves: l'accaparement excessif et abusif de l'espace avec, dans certains cas, non-reconnaissance et remise en question des attributions de pâturages, ou, dans d'autres, attributions fantaisistes de droits de pâturage. Enfin, une meilleure définition juridique des conditions d'octroi de droits de pâturage, dans le respect des règles coutumières, semble nécessaire pour permettre à l'élevage bovin de progresser avec l'aide du crédit.

II faut insister sur la nécessité de se rapprocher, par différentes méthodes (décentralisation, assouplissement et simplification des procédures...), des éleveurs authentiques, motivés et responsables. Cette démarche doit s'accompagner d'un effort de sensibilisation et de formation des éleveurs car le crédit ne peut pas 


\section{P. Lhoste}

s'appuyer sur des projets individualistes et parfois contradictoires. La communauté des éleveurs devra, de plus en plus, prendre en main un certain nombre de décisions qui conditionnent son avenir. Dans le domaine du crédit cela pourrait se traduire par :

- la création de commissions d'éleveurs, compétentes pour l'examen des dossiers, les attributions de terrain, etc. ;

- une organisation collective, qui apparaît nécessaire pour la gestion des pâturages, mais dont les fonctions pourraient s'étendre progressivement: installations communes (sanitaires, zootechniques...), représentativité objective des éleveurs, commercialisation. Cette organisation, certes difficile à promouvoir, conditionne la modernisation souhaitée de l'élevage bovin camerounais, qui doit se faire avec l'ensemble des éleveurs.

\section{CONCLUSION}

II apparaît qu'une politique de crédit à l'élevage bovin traditionnel, aussi fondée soit-elle dans son principe,

LHOSTE (P.) A study of credit to traditional cattle breeders in Cameroon. Revue Elev. Méd. vét. Pays trop., 1990, 43 (1) : 111117.

A credit programme for traditional cattle breeders of Cameroon was set up from 1975 to 1986 in order to favour the modernization of cattle farming. It was evaluated in 1986 that this programme encountered many difficulties and did not reach the expected objectives. It seems important to clarify the legal status of pasture areas and to make the breeder groups aware of their responsabilities in front of such a programme. Key words : Cattle - Breeder - Credit - Traditional farming - Cameroon. est en réalité difficile à mettre en oeuvre chez les éleveurs authentiques.

L'exemple camerounais illustre les risques de dérapage d'un tel projet. L'expérience a en effet prouvé que certains effets pervers d'un crédit mal contrôlé, et plus ou moins détourné de son objectif premier, peuvent compromettre gravement un programme, techniquement fondé, de modernisation de l'élevage traditionnel.

En ce qui concerne les aspects techniques, on peut laisser une grande initiative aux éleveurs, mais il faut impérativement les responsabiliser pour la gestion du crédit et de l'espace. De plus, il est nécessaire de préciser le statut juridique des terres à pâturages en tenant compte des règles coutumières.

\section{REMERCIEMENTS}

Nous tenons à remercier les cadres de la Société d'Études pour le Développement de l'Afrique (SEDA, Yaoundé, Cameroun), et plus particulièrement M.J. LITASSOU pour sa participation aux enquêtes.
LHOSTE (P.). Estudio sobre el crédito accesible a los productores bovinos en Camcrún. Revue Élev. Méd. vét. Pays trop., 1990, 43 (1) : 111-117.

Fue puesto en marcha un programa crédito, accesible a los productores bovinos tradicionales, con el fin de favorizar la modernización de ese sector. Una evaluación efectuada en 1986, evidenció las dificultades encontradas en este proyecto, el cual no alcanzó los objetivos propuestos. En este tipo de programas, es esencial precisar el estatus jurídico de las tierras de pastoreo y responsabilizar a las comunidades de productores. Palabras claves : Bovino - Ganadero - Crédito - Cría tradicional - Camerún.

\section{BIBLIOGRAPHIE}

1. Banque Mondiale. Rapports d'évaluation du premier projet national de développement de l'élevage. Washington, Banque Mondiale, 1978 et 1980.

2. BOUTRAIS (J.). Deux études sur l'élevage en zone tropicale humide (Cameroun). Paris, ORSTOM, 1978.194 p. (Travaux et Documents $n^{\circ} 88$ ).

3. BOUTRAIS (J.). L'élevage soudanien. Des parcours de savanes aux ranchs (Cameroun-Nigeria). Paris, ORSTOM, 1983, 148 p. (Travaux et Documents $n^{\circ} 160$ ). 
4. Commission de contrôle et de coordination du ranching en Adamaoua. Compte rendu de réunion, N'Gaoundéré, Cameroun, Délégation provinciale de l'élevage, décembre 1985.

5. DAWA (O.). Réflexion sur l'avenir du ranching dans la province de l'Adamaoua. N'Gaoundéré, Cameroun, Délégation provinciale de l'élevage, $1986.17 \mathrm{p}$.

6. DOUFISSA (A.). Contribution aux réflexions sur l'avenir de l'élevage et en particulier du ranching en Adamaoua. Rapport final de la commission de contrôle et de coordination des ranchs. N'Gaoundéré, Cameroun, Délégation provinciale de l'élevage, novembre 1985.

7. GTZ. Étude d'aménagement de l'Adamaoua. Eschborn, RFA, GTZ, 1980.

8. GTZ. Rural Finance Sector study (Cameroun). Eschbom, RFA, GTZ, 1986. 92 p.

9. LHOSTE (P.). Étude du crédit aux éleveurs bovins au Cameroun. Maisons-AIfort, IEMVT, 1987. $54 \mathrm{p}$.

10. MOYERSOEN (A.). Adamaoua: Adaptation des financements aux différents modèles de ranchs. N'Gaoundéré, Cameroun, FONADER, $1985.18 \mathrm{p}$.

11. RIPPSTEIN (G.). Étude sur la végétation de l'Adamaoua. Évolution, conservation, régénération et amélioration d'un écosystème pâturé au Cameroun. Maisons-Alfort, IEMVT, 1986. 366 p. (Études et Synthèses n 14 ). 